\title{
Hospitalisations pour blessures intentionnelles dans les régions à fort pourcentage de résidents d'identité autochtone, 2004-2005 à 2009-2010
}

\author{
L. N. Oliver, Ph. D.; P. Finès, Ph. D.; E. Bougie, Ph. D.; D. Kohen, Ph. D.
}

Cet article a fait l'objet d'une évaluation par les pairs.

\section{Résumé}

Introduction : Cette étude décrit les taux d'hospitalisation pour blessures auto-infligées et pour blessures par agression dans les régions à pourcentage relativement élevé de résidents s'identifiant comme membres des Premières nations, Métis ou Inuits, par cause de blessure, par tranche d'âge et par sexe.

Méthodologie : Tous les dossiers de sortie des hôpitaux de soins de courte durée des provinces et territoires du Canada hors Québec ont été tirés de la Base de données sur les congés des patients. Les aires de diffusion où plus de $33 \%$ des résidents ont déclaré appartenir à un groupe autochtone au recensement de 2006 ont été classées comme "régions à fort pourcentage d'Autochtones ".

Résultats : Dans l'ensemble, dans les régions à fort pourcentage d'Autochtones, les taux d'hospitalisation normalisés selon l'âge (THNA) pour blessures auto-infligées étaient plus élevés chez les femmes, tandis que les THNA pour blessures par agression étaient plus élevés chez les hommes. Comparativement aux résidents des régions à faible pourcentage d'Autochtones, les résidents des régions à fort pourcentage d'Autochtones étaient au moins trois fois plus susceptibles d'avoir été hospitalisés pour blessures autoinfligées et au moins cinq fois plus susceptibles d'avoir été hospitalisés pour blessures par agression.

Conclusion : Les recherches à venir devraient analyser les comorbidités et les conditions socioéconomiques ainsi que les comportements à risque individuels comme facteurs associés à l'hospitalisation pour blessures auto-infligées.

Mots-clés : Autochtone, blessures, blessures intentionnelles, blessures auto-infligées, agression

\section{Introduction}

Les Autochtones du Canada (c.-à-d. les membres des Premières nations, les Métis et les Inuits) ont généralement une moins bonne santé et une espérance de vie plus courte que l'ensemble de la population canadienne $^{1-9}$. Ils présentent également des taux plus élevés de mortalité et de morbidité associées aux blessures ${ }^{10-12}$. L'étude des blessures est importante, car elles sont considérées comme largement évitables, ont des répercussions à long terme sur la santé et sont associées à des coûts de santé élevés ${ }^{3,4,13}$. Les blessures intentionnelles - blessures auto-infligées et blessures par agression - revêtent une importance particulière pour la population autochtone, car plusieurs études ont fait ressortir des taux relativement élevés de mortalité attribuable aux suicides et aux homicides chez les Premières nations et les Métis ${ }^{2,5}$. Par exemple, les taux de mortalité attribuable aux suicides et aux blessures auto-infligées chez les résidents de l'Inuit Nunangat, qui sont principalement inuits, sont au moins 11 fois plus élevés que dans le reste du Canada ${ }^{6,7}$.

Bien qu'on utilise en général les données sur la mortalité pour étudier les blessures intentionnelles, les données d'hospitalisation sont également pertinentes car un grand nombre de blessures intentionnelles n'entraînent pas la mort et sont associées à des comorbidités (p. ex. des diagnostics de troubles de santé mentale). Comme exemple de l'importance des données d'hospitalisation, on peut noter que les taux d'hospitalisation pour blessures autoinfligées sont plus élevés chez les femmes ${ }^{8,14,15}$, alors que les données sur la mortalité indiquent des taux de suicide plus élevés chez les hommes ${ }^{15,16}$.

Les indicateurs d'identité autochtone ne sont pas systématiquement accessibles dans les bases de données nationales sur les hospitalisations qui contiennent l'information sur les blessures. Par conséquent, les études reposent soit sur des bases de données provinciales contenant une information identificatrice, soit sur une approche géographique. Les études provinciales fondées sur des données d'hospitalisation contenant des indicateurs d'identité autochtone concernent seulement les provinces de l'Ouest, qui disposent d'information sur les personnes inscrites en vertu de la Loi sur les Indiens. Ces études ont fait ressortir des taux plus élevés d'hospitalisation pour blessures autoinfligées et pour blessures par agression

Rattachement des auteurs :

Division de l'analyse de la santé, Statistique Canada, Ottawa (Ontario), Canada

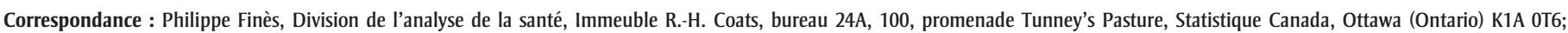
tél. : 514-283-6847; téléc. : 514-283-9350; courriel : philippe.fines@statcan.gc.ca 
chez les membres inscrits des Premières nations comparativement à l'ensemble de la population ${ }^{1,8,9}$. Cependant, ces études n’englobaient ni les Métis, ni les Inuits, ni les membres non inscrits des Premières nations.

Les études reposant sur une approche géographique ont fait ressortir des taux élevés d'hospitalisation pour blessures intentionnelles dans les régions à fort pourcentage d'Autochtones. Une étude d'envergure nationale (excluant le Québec) a montré que, dans les régions à prédominance autochtone, les taux d'hospitalisation pour blessures par agression et pour blessures auto-infligées étaient respectivement neuf et six fois plus élevés que dans les régions à faible pourcentage d'Autochtones ${ }^{10}$. En Ontario, les hospitalisations à la suite d'une agression étaient au moins 17 fois plus nombreuses, et les taux de blessures auto-infligées étaient au moins 6 fois plus élevés dans les collectivités des Premières nations que dans les municipalités du sud de la province ${ }^{11}$. Il existe relativement peu de données sur les hospitalisations pour blessures intentionnelles dans la population inuite, mais des taux élevés de crimes violents, de suicides et d'hospitalisations pour blessures non intentionnelles ont été observés dans des collectivités inuites ${ }^{6,7,17}$. On a récemment fait état de lacunes à propos des blessures dans la recherche en santé chez les Métis ${ }^{12}$. Bien qu'aucune étude n'ait été trouvée concernant les hospitalisations pour blessures auto-infligées ou pour blessures par agression dans la population métisse, une étude récente a révélé que des idées suicidaires étaient plus fréquemment déclarées chez les Métis que chez les non-Autochtones ${ }^{18}$.

Notre analyse, qui est fondée à la fois sur des données nationales et sur une approche géographique, porte sur les hospitalisations pour blessures intentionnelles chez les enfants et les adultes vivant dans les régions où au moins $33 \%$ des résidents ont déclaré appartenir à un groupe autochtone. Nous avons visé deux objectifs : d'une part, établir les taux d'hospitalisation, par tranche d'âge et par sexe, pour blessures auto-infligées et pour blessures par agression dans les régions à proportion relativement élevée de membres des Premières nations, de Métis et
d'Inuits et, d'autre part, comparer ces taux avec ceux des régions à proportion relativement faible d'Autochtones. Notre étude se distingue des autres ${ }^{10,17,19}$ car elle traite des blessures intentionnelles à l'échelle nationale, examine différentes causes de blessure et compare les régions à fort pourcentage aux régions à faible pourcentage d'Autochtones.

\section{Méthodologie}

Nous utilisons ici la même base de données et la même méthodologie que pour une étude antérieure sur les hospitalisations pour blessures non intentionnelles ${ }^{20}$.

\section{Données d'hospitalisation}

Les données d'hospitalisation relatives à six années financières (2004-2005 à 20092010) ont été tirées de la Base de données sur les congés des patients ${ }^{21}$. Ce fichier contient des données sur toutes les sorties des patients des hôpitaux de soins de courte durée (congés, décès, départs volontaires et transferts) de l'ensemble des provinces et territoires du Canada à l'exclusion du Québec. Pour chaque sortie, nous disposons de données sur l'âge, sur le sexe, sur le code postal de résidence, sur les dates d'admission et de sortie et sur les codes de diagnostic correspondant à la Classification statistique internationale des maladies et des problèmes de santé connexes, Dixième version, Canada (CIM-10$\mathrm{CA})^{22}$.

Nous avons examiné deux catégories de blessures intentionnelles : les blessures auto-infligées (faites délibérément à soimême) et les blessures par agression (infligées délibérément par une autre personne, mais excluant les blessures de guerre et les blessures dues à une intervention par les forces de l'ordre). Les blessures auto-infligées ont été classées en coupure ou perforation, noyade ou suffocation et empoisonnement. Les blessures par agression ont quant à elles été classées en coupure ou perforation, heurts ou coups et agression sexuelle ou maltraitance. Les autres catégories de blessures auto-infligées et de blessures par agression (chutes, blessures par le feu ou par substances brûlantes, blessures par arme à feu, accidents de transport terrestre, etc.) n’ont pas été analysées en raison de la faible taille des échantillons. Toutes les catégories, hormis celle des agressions sexuelles et de la maltraitance, correspondent aux codes de la CIM utilisés dans le système International Collaborative Effort on Injury Statistics. Des exemples de chaque catégorie de blessure intentionnelle peuvent être obtenus sur demande.

Les personnes transférées d'un établissement à un autre ont plusieurs dossiers de sortie pour un même épisode de blessure, de sorte qu'une blessure pourrait être comptée plus d'une fois. Pour éviter les comptages multiples, nous avons considéré que toute personne ayant reçu son congé d'un établissement et ayant été admise dans un autre établissement le même jour constituait un seul épisode. Les données renvoient aux épisodes de blessure plutôt qu'au nombre de personnes blessées, car une même personne a pu être hospitalisée plus d'une fois au cours des six ans ciblés par l'étude. Enfin, certains épisodes de blessures multiples pourraient être comptabilisés dans plusieurs catégories (p. ex. empoisonnement et coupure/perforation dans le cas d'une blessure auto-infligée).

\section{Méthode des géozones}

Les aires de diffusion (AD) sont de petites unités géographiques regroupant de 400 à 700 personnes $^{23}$. Les AD dont plus de $33 \%$ des résidents ont déclaré appartenir à un groupe autochtone au recensement de 2006 ont été classées comme régions à fort pourcentage d'Autochtones, puis réparties selon le groupe d'identité autochtone prédominant (Premières nations, Métis ou Inuits). Cette méthode, déjà utilisée ailleurs ${ }^{10,17}$, a été décrite dans un autre $\operatorname{article}^{24}$. Nous avons utilisé les codes postaux résidentiels figurant dans les dossiers de sortie pour déterminer l'AD de résidence en utilisant le Fichier de conversion des codes postaux plus ${ }^{25}$.

En 2006, on comptabilisait au Canada (hors Québec) 38369 AD avec moins de $33 \%$ de résidents ayant déclaré appartenir à un groupe autochtone, ce qui en faisait des régions à faible pourcentage d'Autochtones. Des $2174 \mathrm{AD}$ où plus de 
$33 \%$ des résidents avaient déclaré appartenir à un groupe autochtone, 1929 comptaient un fort pourcentage de membres des Premières nations, 186 un fort pourcentage de Métis et 59 un fort pourcentage d'Inuits.

Ces quatre types de régions - c'est-à-dire celles à fort pourcentage de membres des Premières nations, de Métis ou d'Inuits et celles à faible pourcentage d'Autochtones - différaient les unes des autres sur le plan des caractéristiques socioéconomiques. Comme l'illustre le tableau 1, les $\mathrm{AD}$ à faible pourcentage d'Autochtones sont généralement plus riches que les $\mathrm{AD}$ à fort pourcentage d'Autochtones.

\section{Analyses}

Nous avons calculé les taux d'hospitalisation normalisés selon l'âge (THNA) pour la période de 6 ans à l'étude (2004-2005 à 2009-2010) et les avons normalisés en fonction de la structure par âge de la population autochtone en 2006 par tranches d'âge de 5 ans (c.-à-d. qu'ils correspondent à la somme pondérée des taux d'hospitalisation par âge, la pondération se faisant en fonction de la proportion de chaque tranche d'âge dans la structure par âge de la population de référence). Le dénominateur employé pour calculer les taux a été tiré du recensement de 2006 et correspond au point médian des données d'hospitalisation, qui a été multiplié par 6 afin d'établir les THNA pour l'ensemble des 6 ans. Des THNA (pour 10000 personnes-années) ont été établis pour cinq tranches d'âge (0-9, 10-19, 20-29, 30-44 et 45 ans et plus) et pour les deux sexes pour les régions à fort pourcentage de membres des Premières nations, de Métis ou d'Inuits et pour les régions à faible pourcentage d'Autochtones. Comme les hospitalisations pour blessures autoinfligées étaient rares chez les enfants de 9 ans et moins, nous ne les avons pas incluses dans l'étude. Nous ne disposions pas des données détaillées sur l'âge et le sexe nécessaires à la normalisation selon l'âge pour un petit nombre d'AD en raison de la faible taille de leur population, du taux global de non-réponse ou du dénombrement incomplet des réserves indiennes. Dans ces cas, l'âge et le sexe ont été estimés à partir des effectifs d'ensemble de la population ou à partir d'estimations pour la population des réserves indiennes dénombrées de façon incomplète. Les rapports de taux permettent de comparer les taux des régions à fort pourcentage de membres des Premières nations, de Métis ou d'Inuits avec ceux des régions à faible pourcentage d'Autochtones. Les intervalles de confiance (IC) pour les taux et les rapports de taux sont fondés sur une hypothèse de log-normalité établie selon la méthode de $\mathrm{Kegler}^{26}$. On a jugé que deux taux ou rapports de taux étaient significatifs lorsque leurs IC à $95 \%$ ne se chevauchaient pas. Toutes les analyses ont été effectuées au moyen du logiciel d'analyse statistique SAS, version 9.1.3 (SAS Institute Inc., Cary, Caroline du Nord, États-Unis).

\section{Résultats}

Le tableau 2 présente les statistiques descriptives concernant l'échantillon et le nombre d'hospitalisations pour blessures auto-infligées et pour blessures par agression. Les femmes ont été plus susceptibles

TABLEAU 1

Caractéristiques démographiques et socioéconomiques au recensement de 2006, par identité autochtone prédominante de l'aire de diffusion, Canada (à l'exception du Québec)

\begin{tabular}{|c|c|c|c|c|}
\hline & $\begin{array}{l}\text { AD à fort pourcentage } \\
\text { de membres des } \\
\text { Premières nations }\end{array}$ & $\begin{array}{l}\mathrm{AD} \text { à fort } \\
\text { pourcentage } \\
\text { de Métis }^{\mathrm{a}}\end{array}$ & $\begin{array}{l}\text { AD à fort } \\
\text { pourcentage } \\
\text { d'Inuits }^{\mathrm{a}}\end{array}$ & $\begin{array}{c}\text { AD à faible } \\
\text { pourcentage } \\
\text { d'Autochtones }\end{array}$ \\
\hline Nombre d'AD (n) & 1288 & 178 & 56 & 38710 \\
\hline Population d'identité autochtone (\%) & 79,9 & 54,7 & 81,4 & 2,8 \\
\hline Population vivant dans des logements surpeuplés (\%) & 19,7 & 8,1 & 27,4 & 3,2 \\
\hline Population de 25 à 64 ans sans diplôme d'études secondaires (\%) & 42,1 & 32,6 & 41,5 & 14,4 \\
\hline Population âgée de 15 ans et plus sans emploi (\%) & 20,0 & 12,3 & 16,5 & 6,2 \\
\hline Population âgée de 15 ans et plus sur le marché du travail (\%) & 55,5 & 63,6 & 66,3 & 67,7 \\
\hline$A D$ relevant d'une $R M R / A R$ & 21,8 & 27,4 & 0,0 & 78,9 \\
\hline AD relevant d'une ZIM forte/modérée ${ }^{b}$ & 6,8 & 14,0 & 0,0 & 11,8 \\
\hline
\end{tabular}

Source : Recensement de 2006.

Abréviations : $\mathrm{AD}$, aire de diffusion; RMR/AR, région métropolitaine de recensement/agglomération de recensement; ZIM, zone d'influence métropolitaine.

Remarque : Ce tableau ne concerne que les $\mathrm{AD}$ pour lesquelles les caractéristiques à l'étude étaient connues.

${ }^{a}$ Les $\mathrm{AD}$ dont au moins $33 \%$ de la population a déclaré appartenir à un groupe autochtone ont été classées comme régions à fort pourcentage de résidents d’identité autochtone. La classification d'une région selon le fort pourcentage de membres des Premières nations, de Métis ou d'Inuits est fondée sur le groupe d'identité autochtone prédominant.

${ }^{b}$ Les ZIM ont servi à classer les municipalités à l'extérieur d'une RMR/AR en fonction du pourcentage de la population active se déplaçant dans une RMR/AR pour aller travailler. 


\section{TABLEAU 2}

Dénombrement et statistiques descriptives des hospitalisations pour blessures intentionnelles, par sexe, par identité autochtone prédominante de l'aire de diffusion, par tranche d'âge et par principale cause de blessure, Canada (à l'exception du Québec), 2004-2005 à 2009-2010

\begin{tabular}{|c|c|c|c|c|}
\hline \multirow[t]{2}{*}{ Statistiques descriptives } & \multicolumn{2}{|c|}{$\begin{array}{c}\text { Blessures } \\
\text { auto-infligées }\end{array}$} & \multicolumn{2}{|c|}{$\begin{array}{c}\text { Blessures } \\
\text { par agression }\end{array}$} \\
\hline & Nombre & $\%$ & Nombre & $\%$ \\
\hline Total (toutes causes confondues) & 77497 & & 43854 & \\
\hline \multicolumn{5}{|l|}{ Sexe } \\
\hline Femmes & 47341 & 61,1 & 8376 & 19,1 \\
\hline Hommes & 30156 & 38,9 & 35478 & 80,9 \\
\hline \multicolumn{5}{|l|}{ Identité autochtone prédominante de l'AD } \\
\hline Fort pourcentage de membres des Premières nations & 5424 & 7,0 & 7682 & 17,5 \\
\hline Fort pourcentage de Métis & 622 & 0,8 & 774 & 1,8 \\
\hline Fort pourcentage d'Inuits & 781 & 1,0 & 480 & 1,1 \\
\hline Faible pourcentage d'Autochtones & 70670 & 91,2 & 34918 & 79,6 \\
\hline \multicolumn{5}{|l|}{ Tranche d'âge (ans) } \\
\hline 0 à 9 & $\mathrm{x}$ & $\mathrm{x}$ & 1272 & 2,9 \\
\hline 10 à 19 & 13545 & 17,5 & 7189 & 16,4 \\
\hline 20 à 29 & 16156 & 20,8 & 14505 & 33,1 \\
\hline 30 à 44 & 23837 & 30,8 & 12715 & 29,0 \\
\hline 45 et plus & 23931 & 30,9 & 8173 & 18,6 \\
\hline \multicolumn{5}{|l|}{ Principale cause de blessure } \\
\hline Empoisonnement & 66724 & 86,1 & - & - \\
\hline Coupure ou perforation & 6956 & 9,0 & 8917 & 20,3 \\
\hline Noyade ou suffocation & 1464 & 1,9 & - & - \\
\hline Heurts ou coups & - & - & 26211 & 59,8 \\
\hline Agression sexuelle ou maltraitance & s.o. & s.o. & 3108 & 7,1 \\
\hline
\end{tabular}

Source : Base de données sur les congés des patients, 2004-2005 à 2009-2010.

Abréviation : $\mathrm{AD}$, aire de diffusion.

Remarques : Le symbole « $\mathrm{x} »$ signale que les données doivent être gardées confidentielles en vertu des dispositions de la Loi sur la statistique.

Le symbole «— » signale que les données sont négligeables (faible nombre d’hospitalisations, non analysé en détail).

d'avoir été hospitalisées pour blessures auto-infligées, tandis que les hommes ont été plus susceptibles d'avoir été hospitalisés pour blessures par agression. Les trois causes les plus courantes de blessures auto-infligées étaient les empoisonnements (86 \%), les coupures ou perforations $(9 \%)$ et les noyades ou suffocations (2\%) (tableau 3). Les trois causes les plus courantes de blessures par agression étaient les heurts ou coups (60\%), les coupures ou perforations (20\%) et les agressions sexuelles ou la maltraitance (7 \%) (tableau 4). Les heurts ou coups étaient la cause la plus fréquente dans chaque type de région et chez les deux sexes, la deuxième était les coupures ou perforations chez les hommes et les agressions sexuelles ou la maltraitance chez les femmes.

\section{Hospitalisations pour blessures auto- infligées}

Dans toutes les régions (tant à fort qu'à faible pourcentage d'Autochtones), les THNA pour blessures auto-infligées, toutes causes confondues, étaient significativement plus élevés chez les femmes que chez les hommes (tableau 3), et ce, pour tous les âges comme dans chaque tranche d'âge. Par exemple, les THNA pour toutes les causes et toutes les tranches d'âge confondues allaient chez les femmes de 6,2 pour 10000 personnesannées [IC à $95 \%$ : 6,2 à 6,3] (régions à faible pourcentage d'Autochtones) à 44,0 pour 10000 personnes-années [IC à $95 \%$ : 40,5 à 47,9 ] (régions à fort pourcentage d'Inuits) et chez les hommes de 3,8 [IC à $95 \%: 3,7$ à 3,8 ] (régions à faible pourcentage d'Autochtones) à 21,5 [IC à $95 \%: 19,1$ à 24,2] (régions à fort pourcentage d'Inuits).

Pour toutes les causes et toutes les tranches d'âge confondues, les rapports de taux indiquent que, chez les hommes comme chez les femmes, les THNA pour blessures auto-infligées étaient au moins 2,5 fois plus élevés dans les régions à fort pourcentage de membres des Premières nations, de Métis ou d'Inuits que dans les régions à faible pourcentage d'Autochtones (figure 1). Les rapports de taux les plus élevés ont été observés chez les hommes comme chez les femmes dans les régions à fort pourcentage d'Inuits, avec des THNA pour blessures auto-infligées de plus de 5 fois plus élevés que dans les régions à faible pourcentage d'Autochtones.

Les THNA pour blessures auto-infligées culminaient en général dans la tranche d'âge des 20 à 29 ans chez hommes comme chez les femmes, mais ce pic lié à l'âge n'était statistiquement significatif que dans les régions à fort pourcentage de membres des Premières nations. La tranche d'âge des 45 ans et plus est celle où les THNA pour blessures auto-infligées étaient les moins élevés, et ce dans chaque type de région. De même, les rapports de taux étaient moins élevés dans la tranche d'âge des 45 ans et plus que dans les trois tranches d'âge plus jeunes étudiées, tendance qui a été observée dans les régions à fort pourcentage de membres des Premières nations, de Métis ou d'Inuits (données non présentées).

\section{Blessures auto-infligées par empoisonnement, par coupure ou perforation et par noyade ou suffocation}

Les types de blessures auto-infligées variaient selon le sexe et le groupe d'identité prédominant dans la région (tableau 3 ). Chez les femmes, la majorité des hospitalisations pour blessures auto-infligées étaient survenues à la suite d'un empoisonnement et leur proportion était similaire dans les régions à fort et à faible 


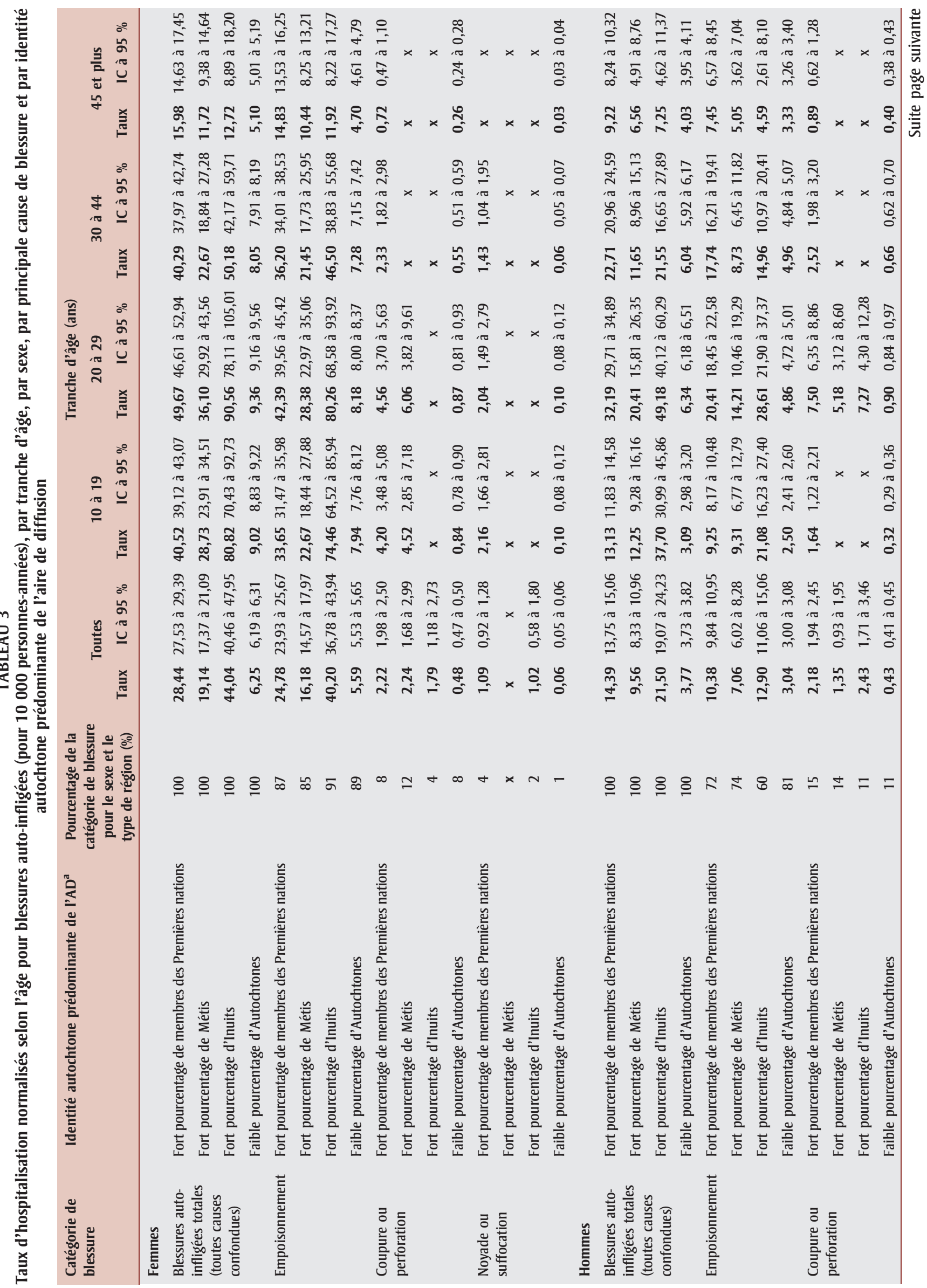









pourcentage d'Autochtones (entre $85 \%$ et $91 \%)$. La deuxième cause d'hospitalisation pour blessures auto-infligées chez les femmes était les coupures ou les perforations, avec des proportions variant entre $4 \%$ (régions à fort pourcentage d'Inuits) et $12 \%$ (régions à fort pourcentage de Métis). Le pourcentage d'hospitalisations pour blessures auto-infligées à la suite d'une noyade ou d'une suffocation était inférieur à $4 \%$ chez les femmes dans toutes les régions. Chez les hommes, la majorité des hospitalisations pour blessures auto-infligées étaient aussi attribuables à un empoisonnement, dans une proportion variant entre $60 \%$ (régions à fort pourcentage d'Inuits) et $81 \%$ (régions à faible pourcentage d'Autochtones). Le pourcentage d'hospitalisations survenues à la suite d'une coupure ou d'une perforation variait entre $11 \%$ et $15 \%$, et le pourcentage d'hospitalisations survenues à la suite d'une noyade ou d'une suffocation variait entre $3 \%$ (régions à faible pourcentage d'Autochtones) et $9 \%$ (régions à fort pourcentage d'Inuits).

On a pu observer de grandes disparités entre les régions à fort pourcentage d'Autochtones et celles à faible pourcentage d'Autochtones pour certaines causes de blessures auto-infligées. Plus précisément, et bien que les taux globaux aient été faibles, les hommes et les femmes vivant dans une région à fort pourcentage de membres des Premières nations ou d'Inuits étaient au moins 9 fois plus susceptibles d'avoir été hospitalisés pour blessures auto-infligées à la suite d'une noyade ou d'une suffocation que les personnes vivant dans les régions à faible pourcentage d'Autochtones (figure 1). De plus, certains rapports de taux d'hospitalisation pour blessures auto-infligées à la suite d'une coupure ou d'une perforation étaient plus élevés que le rapport de taux d'hospitalisation toutes causes confondues, notamment dans le cas des hommes vivant dans les régions à fort pourcentage de membres des Premières nations (rapport de taux : 5,1 contre 3,8) et dans le cas des femmes vivant dans les régions à fort pourcentage de Métis (rapport de taux : 4,6 contre 3,1 ). Les femmes vivant dans les régions à fort pourcentage d'Inuits ont présenté un rapport de taux d'hospitalisation pour coupures et perforations moins élevé que le rapport de taux d'hospitalisation global pour blessures auto-infligées (rapport de taux : 3,7 contre 7,1). En ce qui concerne les empoisonnements autoinfligés, le rapport de taux chez les hommes dans les régions à fort pourcentage d'Inuits était inférieur au rapport de taux global concernant les blessures auto-infligées (rapport de taux : 4,2 contre 5,7). Enfin, les rapports de taux concernant les empoisonnements auto-infligés étaient plus élevés chez les femmes que chez les hommes dans les régions à fort pourcentage de membres des Premières nations et d'Inuits.

\section{Hospitalisations pour blessures par agression}

Les THNA pour blessures par agression étaient plus élevés chez les hommes que

\section{FIGURE 1}

Rapports de taux pour les blessures auto-infligées, toutes tranches d'âge confondues, par sexe, par principale cause de blessure et par identité autochtone prédominante de l'aire de diffusion, Canada (à l'exception du Québec), 2004-2005 à 2009-2010

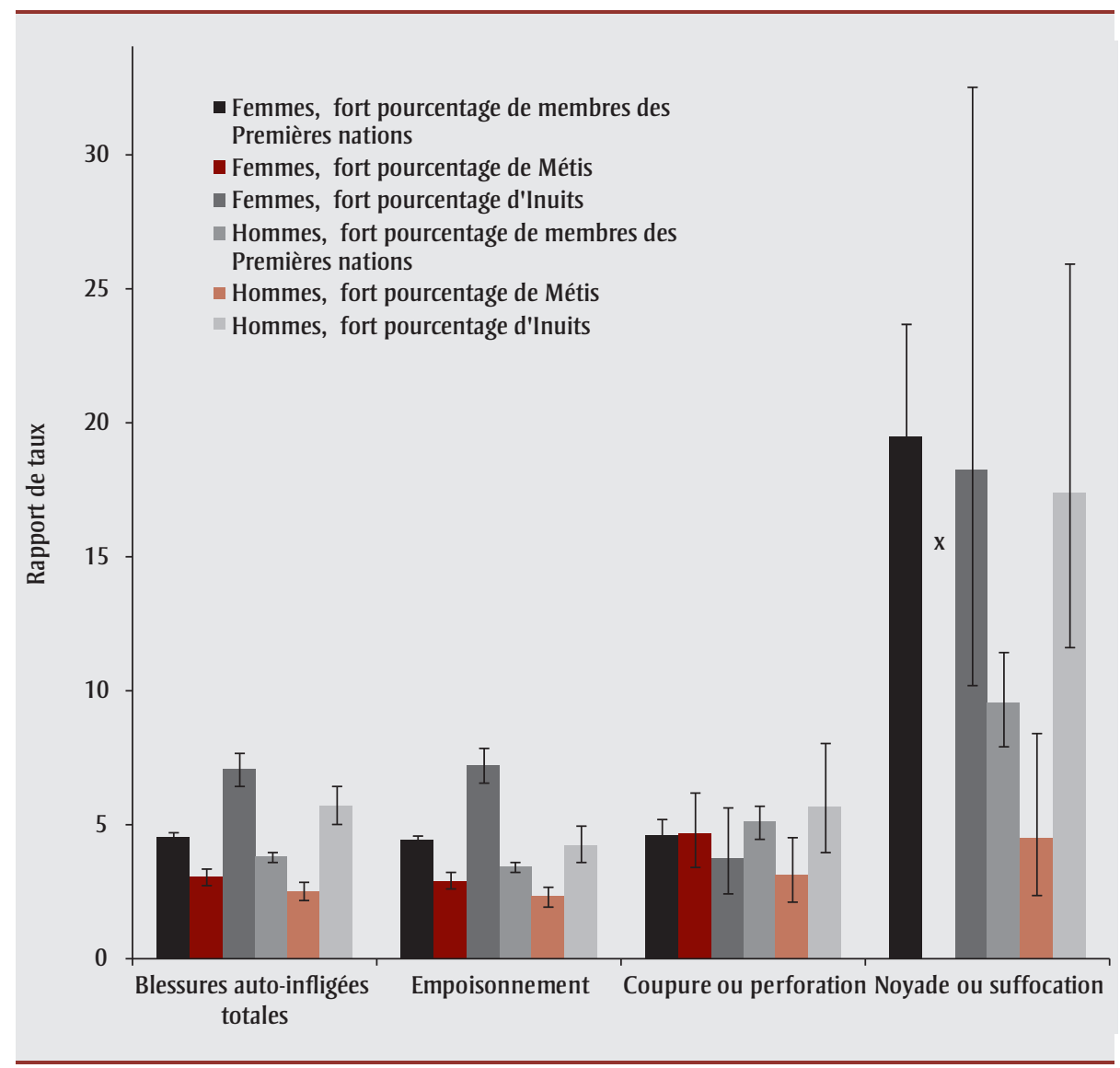

Remarques : Le groupe de référence est constitué des régions à faible pourcentage d'Autochtones.

Le symbole « $\mathrm{x}$ » signale que les données doivent être gardées confidentielles en vertu des dispositions de la Loi sur la statistique.

Tous les rapports de taux sont statistiquement significatifs puisque leur intervalle de confiance ne contient pas la valeur 1 . 
FIGURE 2

Rapports de taux pour les blessures par agression, toutes tranches d'âge confondues, par sexe, par principale cause de blessure et par identité autochtone prédominante de l'aire de diffusion, Canada (à l'exception du Québec), 2004-2005 à 2009-2010



Remarques : Le groupe de référence est constitué des régions à faible pourcentagé d’Autochtones.

Tous les rapports de taux sont statistiquement significatifs puisque leur intervalle de confiance ne contient pas la valeur 1 .

Premières nations, de Métis ou d’Inuits, étaient plus élevés chez les femmes que chez les hommes. Les femmes vivant dans les régions à fort pourcentage de membres des Premières nations étaient 17,9 fois plus susceptibles d'avoir été hospitalisées à la suite d'une agression que celles vivant dans les régions à faible pourcentage d'Autochtones et celles vivant dans les régions à fort pourcentage de Métis et d'Inuits étaient respectivement 9,4 et 19,2 fois plus susceptibles d'avoir été hospitalisées à la suite d'une agression. Chez les hommes, les taux d'hospitalisation pour blessures par agression étaient, comparativement aux régions à faible pourcentage d'Autochtones, 9,7 fois plus élevés dans les régions à fort pourcentage de membres des Premières nations, 6,0 fois plus élevés dans celles à fort pourcentage de Métis et 5,0 fois plus élevés dans celles à fort pourcentage d'Inuits (figure 2).
Comme dans le cas des blessures autoinfligées, les THNA pour blessures par agression culminaient dans la tranche d'âge des 20 à 29 ans, chez les hommes comme chez les femmes et dans toutes les régions (données non présentées). Les rapports de taux comparant les hospitalisations à la suite d'une agression dans les régions à fort pourcentage d'Autochtones et dans les régions à faible pourcentage d'Autochtones n'ont cependant fait ressortir aucune tendance nette pour l'ensemble des tranches d'âge (données non présentées).

\section{Agressions par heurts ou coups, par coupure ou perforation et par agression sexuelle ou maltraitance}

La majorité des hospitalisations pour blessures par agression étaient dues à des heurts ou coups, aussi bien chez les hommes que chez les femmes, et dans toutes les régions (tableau 4). Les rapports de taux montrent que les hospitalisations pour blessures par coups étaient, pour l'ensemble des tranches d'âge, plus de 20 fois plus nombreuses chez les femmes vivant dans les régions à fort pourcentage de membres des Premières nations et d'Inuits que chez celles vivant dans les régions à faible pourcentage d'Autochtones (figure 2). Le rapport de taux était quelque peu plus faible pour les femmes vivant dans les régions à fort pourcentage de Métis. Chez les hommes, les hospitalisations pour blessures par coups étaient environ 10 fois plus nombreuses dans les régions à fort pourcentage de membres des Premières nations et environ 5 fois plus nombreuses dans les régions à fort pourcentage d'Inuits et de Métis que dans les régions à faible pourcentage d'Autochtones.

Les rapports de taux ont révélé que, chez les hommes, les hospitalisations pour coupures ou perforations à la suite d'une agression étaient environ 10 fois plus nombreuses dans les régions à fort pourcentage de membres des Premières nations, 7 fois plus nombreuses dans celles à fort pourcentage de Métis et 4 fois plus nombreuses dans celles à fort pourcentage d'Inuits que dans celles à faible pourcentage d'Autochtones. Chez les femmes, bien que les THNA pour ces types de blessures par agression aient été faibles - moins de 2 pour 10000 personnes-années dans l'ensemble des régions, que le pourcentage d'Autochtones y soit fort ou faible (tableau 4) -, les hospitalisations pour coupures ou perforations à la suite d'une agression étaient, pour l'ensemble des tranches d'âge, respectivement 17, 10 et 15 fois plus élevées dans les régions à fort pourcentage de membres des Premières nations, de Métis et d'Inuits que dans les régions à faible pourcentage d'Autochtones (figure 2).

Contrairement aux autres types de blessures par agression, les agressions sexuelles et la maltraitance ont été associées à des taux d'hospitalisation plus élevés chez les femmes que chez les hommes dans l'ensemble des régions, que le pourcentage d'Autochtones soit fort ou faible. Les 
rapports de taux indiquent néanmoins que les hommes comme les femmes vivant dans les régions à fort pourcentage de membres des Premières nations, de Métis ou d'Inuits étaient au moins 5 fois plus susceptibles d'avoir été hospitalisés à la suite d'une agression sexuelle ou d'une maltraitance que les personnes vivant dans les régions à faible pourcentage d'Autochtones (figure 2).

\section{Analyse}

Cette étude est importante, car elle est la première à utiliser des données d'hospitalisation à l'échelle de la population canadienne pour décrire les tendances touchant les hospitalisations pour blessures intentionnelles dans les régions à fort pourcentage de membres des Premières nations, de Métis ou d'Inuits et à les comparer aux régions à faible pourcentage d'Autochtones. L'utilisation de données d'hospitalisation sur six ans nous a permis d'établir des taux par cause de blessure intentionnelle, par âge et par sexe, dans les régions à fort et celles à faible pourcentage d'Autochtones. Ces données, essentielles à l'établissement d'un portrait détaillé des différences dans les taux de blessures intentionnelles, peuvent être utilisées également pour en suivre l'évolution. Dans l'ensemble, les taux d'hospitalisation pour blessures auto-infligées dans les régions à fort pourcentage de membres des Premières nations, de Métis ou d'Inuits étaient au moins 3 fois plus élevés que ceux observés dans les régions à faible pourcentage d'Autochtones (quels que soient le sexe, la cause de blessure et l'âge), tandis que les taux d'hospitalisation pour blessures par agression dans les régions à fort pourcentage d'Autochtones étaient au moins 5 fois plus élevés que ceux observés dans les régions à faible pourcentage d'Autochtones (quels que soient le sexe, la cause de blessure et l'âge). Ces observations sont cohérentes avec celles d'autres études fondées sur des données nationales, provinciales et individuelles ${ }^{1,8-11}$. Bien qu'une étude récente, elle aussi fondée sur des données d'hospitalisation, ait indiqué que les taux de blessures autoinfligées étaient plus élevés dans les quartiers à faible statut socioéconomique ${ }^{27}$, notre étude ne permet pas de séparer ces facteurs. Les $\mathrm{AD}$ à fort pourcentage de membres des Premières nations, de Métis ou d'Inuits étant socioéconomiquement défavorisées par rapport aux $\mathrm{AD}$ à faible pourcentage d'Autochtones, l'influence des conditions socioéconomiques sur les taux d'hospitalisation pour blessures dans les régions à fort et à faible pourcentage d'Autochtones est donc un sujet de recherche qu'il serait important d'approfondir.

Les hospitalisations à la suite d'une agression étaient 5 à 10 fois plus nombreuses chez les hommes vivant dans les régions à fort pourcentage d'Autochtones que chez ceux vivant dans les régions à faible pourcentage d'Autochtones. Les femmes vivant dans les régions à fort pourcentage de membres des Premières nations et d'Inuits présentaient un risque particulièrement élevé d'hospitalisation à la suite d'une agression, soit 18 fois plus grand que chez celles vivant dans les régions à faible pourcentage d'Autochtones. Les rapports de taux d'hospitalisation les plus élevés chez les femmes étaient associés aux blessures par heurts ou coups, et les femmes vivant dans les régions à fort pourcentage de membres des Premières nations et d'Inuits étaient 20 fois plus nombreuses à avoir été hospitalisées que celles vivant dans les régions à faible pourcentage d'Autochtones. Des observations semblables ont été faites concernant les femmes dans les collectivités des Premières nations de l'Ontario ${ }^{11}$ et dans les collectivités aborigènes du Queensland (Australie) $^{28}$.

Dans cette étude, nous avons observé que les taux d'hospitalisation pour blessures auto-infligées étaient plus élevés dans les régions à fort pourcentage d'Autochtones et que les empoisonnements, les coupures ou perforations et les noyades ou suffocations étaient les trois principales causes d'hospitalisation. Les taux d'hospitalisation pour blessures auto-infligées étaient plus élevés chez les femmes que chez les hommes, et ils étaient aussi particulièrement élevés chez les femmes comme chez les hommes dans les régions à fort pourcentage d'Inuits. Ces observations sont cohérentes avec celles d'autres études menées au Canada ${ }^{1,6,7,15}$ et à l'étranger (Nouvelle-Zélande, Alaska, Groenland, Norvège ${ }^{29,30}$, de même qu'avec celles d'une étude sur les idées suicidaires déclarées, qui a révélé que la prévalence des idées suicidaires et des tentatives de suicide était plus élevée chez les personnes s'identifiant comme Métis que chez les répondants non autochtones ${ }^{18}$.

\section{Points forts et points faibles}

Les résultats de cette étude étant présentés par zones géographiques, les associations observées devraient donc être interprétées comme applicables à des zones géographiques seulement.

Bien que l'une des forces de l'étude ait été l'utilisation sur six ans de données sur les départs des hôpitaux pour analyser les hospitalisations pour blessures intentionnelles dans les régions à fort et à faible pourcentage d'Autochtones, cette source de données comportait certaines limites. Comme les indicateurs individuels d'identité autochtone n'étaient pas accessibles dans les dossiers des hôpitaux, nous avons utilisé un seuil de $33 \%$ comme critère pour définir les aires de diffusion à fort pourcentage d'Autochtones. Comme les populations de ces régions comprennent à la fois des résidents autochtones et des résidents non autochtones, les résultats ne peuvent pas être généralisés aux Premières nations, aux Métis et aux Inuits du Canada. Les identificateurs géographiques des dossiers de sortie des hôpitaux indiquent le code postal de résidence des patients, mais non le lieu de la blessure. Des données sur le lieu des blessures autodéclarées ont cependant été publiées dans un autre article ${ }^{31}$.

Les données d'hospitalisation concernant le Québec n'étaient pas disponibles. Les données d'hospitalisation présentées ici ne sont pas représentatives de l'ensemble des blessures auto-infligées et des blessures par agression graves ou nécessitant un traitement médical ${ }^{15}$. De plus, l'étude ne prenait pas en compte les données sur les blessures intentionnelles les plus graves (p. ex. suicide, homicide) ayant causé la mort avant l'hospitalisation. Bien que les données utilisées dans le cadre de cette étude n'aient pas permis pas de déterminer si l'intention à l'origine des blessures autoinfligées était l'automutilation ou le suicide, les blessures auto-infligées ont par ailleurs été considérées comme révélatrices d'une 
mauvaise santé mentale et de tendances suicidaires ${ }^{18,29,30,32-40}$.

Les personnes qui se sont présentées aux services des urgences mais qui n'ont pas été hospitalisées ont été exclues. Il est aussi possible que certaines blessures auto-infligées aient été classées comme indéterminées, ce qui entraînerait une sousestimation du taux réel d'hospitalisation pour blessures ${ }^{41}$. Les données d'hospitalisation ne comprenaient pas suffisamment de détails sur le statut socioéconomique et sur le comportement à l'échelle individuelle pour faire ressortir des facteurs de risque individuels d'hospitalisation pour blessures intentionnelles ${ }^{42}$.

\section{Conclusion}

Notre étude a montré que les taux d'hospitalisation pour blessures auto-infligées et pour blessures par agression étaient plus élevés dans les régions à fort pourcentage de résidents d'identité autochtone (c.-à-d. membres des Premières nations, Métis ou Inuits) que dans les régions à faible pourcentage de résidents d'identité autochtone. Cette étude est la première au Canada à aborder les hospitalisations pour blessures intentionnelles par cause selon l'identité autochtone prédominante dans chaque région. À l'avenir, des recherches pourraient porter sur la gravité de ces blessures, car certaines données indiquent que cette gravité pourrait varier entre les populations autochtones et non autochtones ${ }^{9,43}$. Étant donné qu'environ $70 \%$ des personnes hospitalisées pour blessures auto-infligées avaient reçu un diagnostic de maladie mentale $^{15}$, il serait important que les recherches à venir prennent en considération les comorbidités, puisque des troubles de santé mentale ont été associés aux hospitalisations pour blessures autoinfligées $^{36}$. Des recherches pourraient explorer les conditions socioéconomiques dans la région ainsi que les comportements à risque individuels associés aux blessures intentionnelles et aux hospitalisations $^{15,42,44}$. Enfin, l'éclaircissement des facteurs culturels, historiques et environnementaux précis à l'origine des taux plus élevés d'hospitalisation pour blessures autoinfligées et pour blessures par agression dans les régions à fort pourcentage
d'Autochtones $^{36,37,45}$ pourrait contribuer à notre compréhension de la situation.

\section{Remerciements}

Nous remercions John Graham McLean pour sa contribution concernant le suicide. Nous souhaitons également remercier nos deux évaluateurs anonymes pour leurs commentaires judicieux. La présente étude a été financée par la Direction générale de la santé des Premières nations et des Inuits (DGSPNI) de Santé Canada.

\section{Références}

1. Santé Canada. Profil statistique de la santé des Premières nations au Canada. Utilisation des services de santé dans l'Ouest canadien, 2000. Ottawa (Ont.) : Santé Canada; 2009. (nº de catalogue : H34193/4-2008)

2. Tjepkema $M$, Wilkins $R$, Senécal $S$, Guimond E, Penney C. La mortalité chez les Métis et les Indiens inscrits adultes au Canada : étude de suivi sur 11 ans. Rapports sur la santé. 2009;20(4):33-55. (Statistique Canada, n॰ 82-003 au catalogue)

3. Holbrook TL, Hoyt DB, Coimbra R, et al. Trauma in adolescents causes long-term marked deficits in quality of life: adolescent children do not recover preinjury quality of life or function up to two years postinjury compared to national norms. J Trauma. 2007;62(3):577-83.

4. Sauve-qui-pense. Le fardeau économique des blessures au Canada. Toronto (Ont.) : Sauve-qui-pense; 2009.

5. Harrop A, Brant R, Ghali W, Macarthur C. Injury mortality rates in Native and nonNative children: a population-based study. Public Health Rep. 2007;122(3):339-46.

6. Statistique Canada. Tableau 102-0704 Mortalité, selon certaines causes de décès (CIM-10) et le sexe, moyenne de cinq ans, Canada et régions inuites, aux 5 ans, CANSIM [base de données]. Ottawa (Ont.) : Statistique Canada; 2012 [consultation le 12 mars 2012]. Consultable à la page : http://www5.statcan.gc.ca/cansim/a26?id = 1020704 \&pattern $=\& \mathrm{p} 2=49 \& \mathrm{p} 1=1 \&$ tabMode $=$ dataTable \&stByVal $=1 \&$ paSer $=\& \operatorname{csid}=\&$ retrLang $=$ fra\&lang $=$ fra
7. Oliver L, Peters P, Kohen D. Taux de mortalité chez les enfants et les adolescents vivant dans l'Inuit Nunangat, 1994 à 2008. Rapports sur la santé. 2012;23(3);17-23. (Statistique Canada, n 82-003 au catalogue)

8. Colman I, Yiannakoulias N, Schopflocher D, Svenson LW, Rosychuk RJ, Rowe BH. Population-based study of medically treated self-inflicted injuries. CJEM. 2004;6(5): 313-20.

9. Karmali S, Laupland K, Harrop AR, et al. Epidemiology of severe trauma among status Aboriginal Canadians: a populationbased study. CMAJ. 2005;172(8):1007-11.

10. Carrière G, Garner R, Sanmartin C, Équipe de recherche de l'Initiative sur les données longitudinales administratives et sur la santé. Hospitalisations dans des hôpitaux de soins de courte durée et identité autochtone au Canada, 2001-2002. Série de documents de travail de la recherche sur la santé. Ottawa (Ont.) : Statistique Canada; 2010. (Statistique Canada, n॰82-662-X au catalogue, $n^{\circ} 005$ )

11. Fantus D, Shah B, Qiu F, Hux J, Rochon P. Injury in First Nations communities in Ontario. Can J Public Health. 2009;100(4): 258-62.

12. Kumar MB, Wesche S, McGuire C. Trends in Métis-related health research (19802009): Identification of research gaps. Can J Public Health. 2012;103(1):23-8.

13. Institut canadien d'information sur la santé (ICIS). Sommaire : Document sur la qualité des données, Base de données sur les congés des patients, 2006-2007. Ottawa (Ont.) : ICIS; 2007.

14. Alaghehbandan R, Sikdar KC, MacDonald D, Collins KD, Rossignol AM. Unintentional injuries among children and adolescents in Aboriginal and non-Aboriginal communities, Newfoundland and Labrador, Canada. Int J Circumpolar Health. 2010;69: 1:61-71.

15. Institut canadien d'information sur la santé (ICIS). Indicateurs de santé 2011. Ottawa (Ont.) : ICIS; 2011. 
16. Statistique Canada. Tableau 102-0551 Décès et taux de mortalité, selon certains groupes de causes, le groupe d'âge et le sexe, Canada, annuel, CANSIM [base de données]. Ottawa (Ont.) : Statistique Canada [consulté le 8 mars 2012]. Consultable à la page : http://www5.statcan.gc.ca/cansim /pick-choisir $? \mathrm{id}=1020551 \& \mathrm{p} 2=33 \&$ retrLang $=$ fra\&lang $=$ fra

17. Oliver LN, Kohen DE. Hospitalisations d'enfants et de jeunes pour blessure non intentionnelle dans les régions ayant un fort pourcentage de résidents d'identité autochtone, 2001-2002 à 2005-2006. Rapports sur la santé. 2012;23(3):7-16. (Statistique Canada, n॰ 82-003 au catalogue)

18. Kumar MB, Walls M, Janz T, Hutchinson P, Turner T, Graham C. Suicidal ideation among Métis adult men and women associated risk and protective factors: findings from a nationally representative survey. Int J Circumpolar Health. 2012;71:18829.

19. Garner R, Carrière G, Sanmartin C. Équipe de recherche sur l'Initiative sur les données longitudinales administratives et sur la santé. La santé des adultes chez les Premières Nations vivant hors réserve, les Inuits, et les Métis au Canada : l'incidence du statut socioéconomique sur les inégalités en matière de santé. Série de documents de travail de la recherche sur la santé. Ottawa (Ont.) : Statistique Canada; 2010. (Statistique Canada, n॰ $82-662-\mathrm{X}$ au catalogue, $\mathrm{n}^{\circ} 004$ )

20. Finès $P$, Bougie E, Oliver LN, Kohen DE. Hospitalisations pour blessures non intentionnelles chez les adultes au Canada, dans les régions à fort pourcentage de résidents d'identité autochtone. Maladies chroniques et blessures au Canada. 2013:33(4):231246.

21. Institut canadien d'information sur la santé (ICIS). Qualité des données de la Base de données sur les congés des patients suite à la première année de la mise en oeuvre de la CIM-10-CA/CCI - Sommaire. Ottawa (Ont.) : ICIS; 2004.

22. Institut canadien d'information sur la santé (ICIS). Classification statistique internationale des maladies et des problèmes de santé connexes - Dixième version, Canada (CIM10-CA). Ottawa (Ont.) : ICIS; 2009.
23. Statistique Canada. Dictionnaire du Recensement de 2006. Ottawa (Ont.) : Statistique Canada; 2010. (Statistique Canada, n॰ 92-566-X au catalogue)

24. Peters P, Oliver L, Carrière G. Géozones : méthode fondée sur la région géographique pour l'analyse des résultats pour la santé. Rapports sur la santé 2012;23(1):55-64. (Statistique Canada, n 82-003 au catalogue)

25. Wilkins R, Peters PA. FCCP + version $5 \mathrm{~K}$ : guide de l'utilisateur : logiciel de codage géographique basé sur les fichiers de conversion des codes postaux de Statistique Canada. Ottawa (Ont.) : Statistique Canada; 2012. (n॰82F0086-XDB au catalogue)

26. Kegler SR. Applying the compound Poisson process model to the reporting of injuryrelated mortality rates. Epidemiol Perspect Innov. 2007;4:1.

27. Institut canadien d'information sur la santé (ICIS). Indicateurs de santé 2013. Ottawa (Ont.) : ICIS; 2013.

28. Macintosh DJ, Pearson J. Patterns of injury in indigenous Australians admitted to Cairns base hospital. Aust J Rural Health. 2003;11(2):99-106.

29. Hatcher S, Sharon C, Collins N Epidemiology of intentional self-harm presenting to four district health boards in New Zealand over 12 months, and comparison with official data. Aust N Z J Psychiatry. 2009;43(7):659-65.

30. Lehti V, Niemela S, Hoven C, Mandell D, Sourander A. Mental health, substance use and suicidal behaviour among young indigenous people in the Arctic: a systematic review. Soc Sci Med. 2009;69(8);1194-1203.

31. Tjepkema M. Les blessures non mortelles chez les Autochtones. Rapports sur la santé. 2005;16(2):9-24. (Statistique Canada, n 82003 au catalogue)

32. Hunter E, Harvey D. Indigenous suicide in Australia, New Zealand, Canada and the United States. Emerg Med. 2002;14(1):14.

33. Brennan S. La victimisation avec violence chez les femmes autochtones dans les provinces canadiennes, 2009. Juristat. Ottawa (Ont.) : Statistique Canada; 2011. (Statistique Canada, n85-002-X au catalogue).
34. Kirmayer LJ, Brass GM, Tait CL. The mental health of Aboriginal peoples: transformations of identity and community. Canadian Journal of Psychiatry. 2000;45(7):607-16.

35. Mignone J, O’Neil J. Social capital and youth suicide risk factors in First Nations communities. Can J Public Health. 2005 Jan-Feb;96 Suppl 1:S51-4.

36. Alcantara C, Gone JP. Reviewing suicide in native American communities: situating risk and protective factors within a transactional-ecological framework. Death Stud. 2007;31(5):457-77.

37. Wexler L, Hill R, Bertone-Johnson E, Fenaughty A. Correlates of Alaska Native Fatal and Nonfatal Suicidal Behaviors 1990-2001. Suicide Life Threat Behav. 2008 June 38(3), p. 311-319.

38. Penney C, Senécal S, Bobet E. Mortalité par suicide dans les collectivités inuites au Canada : taux et effets des caractéristiques des collectivités. Cahiers québécois de démographie. 2009;38(2):311-343.

39. Procter NG. Parasuicide, self-harm and suicide in Aboriginal people in rural Australia: a review of the literature with implications for mental health nursing practice. Int J Nurs Pract. 2005;11:237-41.

40. Kvernmo S, Rosenvinge JH. Self-mutilation and suicidal behaviour in Sami and Norwegian adolescents: prevalence and correlates. Int $\mathrm{J}$ Circumpolar Health. 2009;68(3):235-48.

41. Bethell J, Rhodes AE. Repérer les cas d'automutilation dans les données des services d'urgence. Rapports sur la santé. 2009;20(2):35-43. (Statistique Canada, $n^{\circ}$ 82-003 au catalogue)

42. Boothroyd LJ, Kirmayer LJ, Spreng S, Malus M, Hodgins S. Completed suicides among the Inuit of northern Quebec, 1982-1996: a case-control study. CMAJ. 2001;165(6): 749-55.

43. Wood DS. The validity of injury surveillance system measures of assault: a lesson from the study of violence in Alaska. J Interpers Violence. 2010;25(2):219-41. 
44. Chandler MJ, Lalonde CE. Cultural continuity as a hedge against suicide in Canada's First Nations. Transcult Psychiatry. 1998;35: 191-219.

45. MacNeil MS. An epidemiologic study of Aboriginal adolescent risk in Canada: the meaning of suicide. J Child Adolesc Psychiatr Nurs. 2008;21(1):3-12. 